\title{
Erratum to: Galaxy Alignments: Theory, Modelling \& Simulations
}

\author{
Alina Kiessling ${ }^{1}$ - Marcello Cacciato ${ }^{2}$ - Benjamin Joachimi ${ }^{3}$. Donnacha Kirk ${ }^{3}$. \\ Thomas D. Kitching ${ }^{4}$. Adrienne Leonard ${ }^{3}$. Rachel Mandelbaum ${ }^{5}$. \\ Björn Malte Schäfer ${ }^{6}$. Cristóbal Sifón ${ }^{2} \cdot$ Michael L. Brown ${ }^{7} \cdot$ Anais Rassat ${ }^{8}$
}

Published online: 12 November 2015

(C) Springer Science+Business Media Dordrecht 2015

\section{Erratum to: Space Sci. Rev. (2015) \\ DOI 10.1007/s11214-015-0203-6}

The original article has been updated due to errors introduced in references and references to figures.

The online version of the original article can be found under doi:10.1007/s11214-015-0203-6.

$\triangle$ A. Kiessling

Alina.A.Kiessling@jpl.nasa.gov

1 Jet Propulsion Laboratory, California Institute of Technology, 4800 Oak Grove Drive, Pasadena, CA 91109, USA

2 Leiden Observatory, Leiden University, P.O. Box 9513, 2300 RA, Leiden, The Netherlands

3 Department of Physics and Astronomy, University College London, Gower Street, London WC1E 6BT, UK

4 Mullard Space Science Laboratory, University College London, Holmbury St. Mary, Dorking, Surrey RH5 6NT, UK

5 Department of Physics, McWilliams Center for Cosmology, Carnegie Mellon University, Pittsburgh, PA 15213, USA

6 Zentrum für Astronomie der Universität Heidelberg, Astronomisches Recheninstitut, Philosophenweg 12, 69120 Heidelberg, Germany

7 Jodrell Bank Centre for Astrophysics, School of Physics and Astronomy, University of Manchester, Oxford Road, Manchester M13 9PL, UK

8 Laboratoire d'astrophysique (LASTRO), Ecole Polytechnique Fédérale de Lausanne (EPFL), Observatoire de Sauverny, 1290 Versoix, Switzerland 